\title{
Medikationssicherheit im Home Care Bereich
}

\section{Entwicklung und Pilotierung eines Critical Incident Reporting Systems}

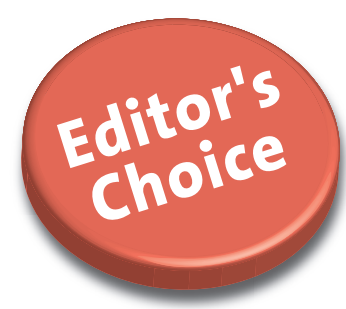

\author{
Carla Meyer-Massetti (Dr. phil. II), Evelyne Krummenacher², Barbara Hedinger-Grogg², \\ Stephan Luterbacher ${ }^{4}$ (Dr. pharm.), Kurt E. Hersberger² (Prof. Dr.)
}

\begin{abstract}
${ }^{1}$ Klinische Pharmazie \& Epidemiologie, Departement Pharmazeutische Wissenschaften, Universität Basel, Schweiz ${ }^{2}$ Pharmaceutical Care Research Group, Departement Pharmazeutische Wissenschaften, Universität Basel, Schweiz ${ }^{3}$ Spitex Stadt Luzern, Schweiz

${ }^{4}$ Dienststelle Gesundheit, Gesundheitsdepartement des Kantons Luzern, Schweiz
\end{abstract}

\begin{abstract}
Zusammenfassung:
Hintergrund: Obwohl medikationsassoziierte Probleme zu den häufigsten unerwünschten Ereignissen im Gesundheitswesen gehören, ist nur wenig über ihre Art und Prävalenz im Home Care Bereich bekannt. Die Verwendung eines Critical Incident Reporting Systems (CIRS) für eine kostengünstige und effiziente Erfassung von Medikationsfehlern zur späteren Trendanalyse ist in der stationären Versorgung etabliert, im ambulanten Bereich aber noch wenig verankert. Empfehlungen zum Format existieren kaum.

Ziel: Das Ziel dieser Arbeit war, ein Fehlermeldesystem spezifisch für die Erfassung der Medikationssicherheit im Home Care Bereich zu realisieren.

Methode: Am Beispiel einer Schweizer Spitex-Organisation wurde ein manuelles CIRS für den Home Care Bereich literaturbasiert entwickelt, pilotiert und implementiert.

Resultate: Das finale Formular lieferte bei guter Akzeptanz durch die Mitarbeitenden und limitiertem Zeitaufwand erste qualitative Hinweise zu Medikationsfehlern im Home Care Umfeld. Am häufigsten erfolgten Meldungen zum Prozessschritt «Richten» (Vorbereiten der Medikamente) in Form von Unterlassung (30\%), falscher Dosierung (17,5\%) und falschem Zeitpunkt (15\%). Als Hauptursache für Fehler gaben die meldenden Personen die Arbeitsbedingungen an (37,9\%), wobei insbesondere Unaufmerksamkeit (68,2\%), Zeitdruck (22,7\%) und Unterbrechungen durch den Klienten (9,1\%) die Auslöser waren.

Schlussfolgerungen: Diese Hinweise waren nützlich für eine Trendanalyse, um zukünftige Interventionen zu planen. Hinsichtlich des Systems ist die Weiterentwicklung zu einem elektronischen CIRS wünschenswert, um den Zeitaufwand für Erfassung und Auswertung zu optimieren und eine nationale Verknüpfung der Fehlermeldungen zu ermöglichen.
\end{abstract}

Schlüsselwörter: ambulante Versorgung, Home Care, Medikationssicherheit, Medikationsfehler, Critical Incident Reporting, CIRS

\section{Medication safety in the home care setting: Development and piloting of a Critical Incident Reporting System}

\section{Abstract:}

Background: While drug-related problems are among the most frequent adverse events in health care, little is known about their type and prevalence in home care in the current literature. The use of a Critical Incident Reporting System (CIRS), known as an economic and efficient tool to record medication errors for subsequent analysis, is widely implemented in inpatient care, but less established in ambulatory care. Recommendations on a possible format are scarce. A manual CIRS was developed based on the literature and subsequently piloted and implemented in a Swiss home care organization.

Aim: The aim of this work was to implement a critical incident reporting system specifically for medication safety in home care. Results: The final CIRS form was well accepted among staff. Requiring limited resources, it allowed preliminary identification and trending of medication errors in home care.

The most frequent error reports addressed medication preparation at the patients' home, encompassing the following errors: omission (30\%), wrong dose (17.5\%) and wrong time (15\%). The most frequent underlying causes were related to working conditions (37.9\%), lacking attention (68.2\%), time pressure (22.7\%) and interruptions by patients $(9.1 \%)$.

Conclusions: A manual CIRS allowed efficient data collection and subsequent analysis of medication errors in order to plan future interventions for improvement of medication safety. The development of an electronic CIRS would allow a reduction of the expenditure of time regarding data collection and analysis. In addition, it would favour the development of a national CIRS network among home care institutions.

Keywords: home care, ambulatory care, medication safety, medication error, critical incident reporting, CIRS 
Was ist (zu dieser Thematik) schon bekannt?

Medikationsfehler sind im Gesundheitswesen häufig. Critical Incident Reporting Systeme (CIRS) zur Erfassung von Medikationsfehlern sind im stationären Bereich weit verbreitet.

\section{Was ist neu?}

Im Rahmen dieser Arbeit wurde ein CIRS zur Erfassung von Medikationsfehlern spezifisch für den Home Care Bereich systematisch entwickelt und pilotiert.

Welche Konsequenzen haben die Ergebnisse für die Pflegepraxis? Die Implementierung eines CIRS erlaubt auch im Home Care Bereich eine kostengünstige, systematische Erfassung von (Beinahe-)Fehlern sowie eine Analyse als Grundlage für nachfolgende Interventionen.

\section{Einleitung}

Das Ausmaß medikationsassoziierter Probleme (unerwünschte Arzneimittelwirkungen/Nebenwirkungen und Medikationsfehler, DRPs) im Home Care Bereich ist bisher wenig bekannt und wird kaum systematisch erfasst. In der aktuellen Fachliteratur wird davon ausgegangen, dass im Gesundheitswesen 30-50\% aller Behandlungsfehler mit der Medikation im Zusammenhang stehen (Meyer-Massetti \& Conen, 2012). In den USA erleben durchschnittlich 5,2\% der Spital-Patienten einen Medikationsfehler, wovon 4,7\% einen Einfluss auf den Outcome haben (Bond \& Raehl, 2007). An der Schnittstelle vom ambulanten zum stationären Bereich verursachen Drug Related Problems (DRPs) in einer aktuellen australischen Studie 2-3\% der Spitaleinweisungen (Roughead, Semple \& Rosenfeld, 2016). Vielen Untersuchungen gemeinsam ist die Annahme, dass Medikationsfehler in mindestens 30-60 \% der Fälle vermeidbar sind (Bates et al., 1995). Angaben aus dem Home Care Bereich von McGraw und Topping (2011) lassen darauf schliessen, dass es sich bei $42 \%$ der medizinischen Fehler um Medikationsfehler handelt (McGraw \& Topping, 2011). In weiteren Studien waren bis zu $30 \%$ der durch Home Care versorgten Patienten einem potenziellen Medikationsfehler ausgesetzt, unter anderem bedingt durch die fragmentierte Versorgung im ambulanten Sektor (Ahrens, 2003; Meredith et al., 2001; Fialova et al., 2005). Während im stationären Bereich insbesondere die Prozessschritte «Verschreibung» und «Richten/Verabreichen» von Medikationsfehlern betroffen sind, ist im Home Care Bereich lediglich bekannt, dass 18-19\% der geriatrischen Patienten in Europa eine ungeeignete Medikation verschrieben wird (Fialova et al., 2005; Fiss, Dreier, Meinke, van den Berg, Ritter \& Hoffmann, 2011).

Durch die Zunahme des Durchschnittsalters der Bevölkerung sowie die Verschiebung der stationären zur ambulanten Behandlung im Rahmen der technischen Entwicklung und der Einführung der Fallpauschalen kommt der Medikationssicherheit im Home Care Bereich wachsende Bedeutung zu.

Die Home Care Organisation Spitex Stadt Luzern betreute auf Non-Profit-Basis mit 230 Angestellten im Jahr 2013 durchschnittlich 870 Klienten, die von 350 niedergelassenen Ärzten und diversen stationären Einrichtungen zugewiesen wurden (Spitex Stadt Luzern, 2013). Eine ihrer häufigsten Aufgaben ist das Richten und Verabreichen von Medikamenten.
Eine 2011 durchgeführte Analyse zeigte einen komplexen Medikationsprozess, der bis zu 20 Schritte umfasst mit durchschnittlich 7,5 \pm 3,5 Medikamenten pro Patient und Tag. Medikationsfehler wurden bisher nur sporadisch und nicht systematisch erfasst. (Meyer-Massetti, Kaiser, Hedinger-Grogg, Luterbacher \& Hersberger, 2012)

Das Gesetz des Kantons Luzern schreibt vor, dass Home Care Organisationen über ein Qualitätsmanagementsystem und damit über Qualitätssicherungsinstrumente verfügen müssen (Heilmittelverordnung des Kantons Luzern vom 28. April 2009, HMV, SRL 830). Da Fehler bei jedem Schritt des Medikationsprozesses auftreten können, ist deren Erfassung zentral als Basis für systematische Auswertungen («Trending») und darauf basierende, gezielte Qualitätsverbesserungsprojekte.

Fehlermeldesysteme sind im Gesundheitswesen als Qualitätserfassungsinstrument weit verbreitet und deren Wert gut etabliert (Shojania, 2008). Die Etablierung eines CIRS wird von der Weltgesundheitsorganisation WHO empfohlen (WHO, 2005). Das freiwillige Sammeln von Daten auf einem standardisierten Papierformular gilt als einfach und kostengünstig (Classen \& Metzger, 2003). Aufgrund der Freiwilligkeit eines CIRS ist die Anzahl der Meldungen in der Regel limitiert, dafür sind keine falschpositiven Meldungen zu erwarten (Tam, Kwok, Fan et al., 2008; Katz \& Lagasse, 2000; Flynn, Barker, Pepper, Bates \& Mikeal, 2002). Die Literatur zeigt, dass im CIRS insbesondere schwerwiegende Zwischenfälle gemeldet werden, was bei limitierten Ressourcen für die Priorisierung von nachfolgenden Medikationssicherheitsaktivitäten wertvoll ist (Akers, Flynn, Davis, Green, Winstead \& Strobel, 2004).

Ziel dieser Arbeit war es, ein Fehlermeldeformular (CIRS-Formular) für die Erfassung von medikationsassoziierten Problemen im Home Care Bereich zu entwickeln und $\mathrm{zu}$ pilotieren sowie dessen Gebrauch mittels einer standardisierten Schulung zu etablieren.

Die Nutzung der patienteneigenen Infrastruktur für das Medikationsmanagement, die komplexe Kommunikation mit mehreren Leistungserbringern und die räumliche Distanz zu den Zuweisern und weiteren Leistungserbringern machen den Medikationsprozess im Home Care Umfeld einzigartig und herausfordernd. Diesen Einflussfaktoren soll bei der Entwicklung eines CIRS-Formulars für die Erfassung von medikationsassoziierten Problemen im Home Care Bereich besondere Beachtung geschenkt werden.

\section{Methoden}

\section{Entwicklung des CIRS-Formulars}

\section{Grundlagen für das Konzept des Formulars - Literaturrecherche}

Es wurde eine Literaturrecherche in den Datenbanken PubMed und Embase (Zeitraum 2001-2014) durchge- 
führt, um ein konkretes Beispiel eines CIRS im Home Care Bereich zu identifizieren.

Verwendet wurde die folgende Freitextsuche: «(CIRS OR critical incident reporting OR incident reporting OR error reporting OR adverse event reporting) AND (home care OR home health OR ambulatory care OR outpatient care OR nursing care)». Die Suche ergab 747 Treffer. Davon bezogen sich 169 Publikationen auf Medikationssicherheit («drug safety» «medication safety»). Deren Abstracts wurden konsultiert: 26 Artikel behandelten Medikationssicherheit im Zusammenhang mit der Verwendung eines CIRS. Diese Artikel wurden nach folgenden Kriterien weiter gescreent:

- Die Studie oder Review bezog sich auf eine ambulante Pflegeorganisation oder ambulante Pflegebetreuung zu Hause

- Die Studie oder Review beschrieb die konkrete Anwendung eines CIRS.

Es wurden 15 Abstracts direkt ausgeschlossen: elf Publikationen waren nicht im ambulanten Pflege-Sektor angesiedelt (vier im Spital resp. auf der Notfallstation, drei in Arztpraxen, eine im Heim, ein klinischer Versuch, eine bei Patienten im ambulanten Bereich ohne Betreuung, eine in mehreren undifferenzierten Sektoren), vier Artikel behandelten das Thema Medikationsfehler als Überblicksartikel ohne konkrete Beispiele von CIRS.

Von elf Publikationen wurde die Volltextversion konsultiert. Zwei Artikel behandelten CIRS im Bereich der Hausarztmedizin, sieben Artikel waren im Home Care Bereich angesiedelt, enthielten aber keinerlei Details zum Aufbau eines CIRS.

Zwei Artikel wurden eingeschlossen: Fernald et al. (2004) nennen konkrete Fragen auf dem CIRS-Formular, McGraw und Topping (2011) geben konkrete Hinweis zu den verwendeten Schadenskategorien.

$\mathrm{Da}$ in der aktuellen Literatur nur wenige Quellen für CIRS im Home Care Bereich verfügbar sind, wurden zusätzlich Spital-CIRS-Formulare aus Spitälern der Schweiz via bilateralem Netzwerk des Schweizerischen Vereins der Amts- und Spitalapotheker GSASA (www.gsasa.ch) als Informationsquelle konsultiert. Eine informelle Umfrage bei weiteren Spitex-Organisationen der Schweiz ergab keine zugänglichen, medikationsspezifischen CIRS-Formulare als Arbeitsgrundlage.

\section{Pretest}

Der auf der Literatur (McGraw \& Topping, 2011; Fernald et al., 2004) und auf Spital-CIRS basierende Formular-Entwurf wurde einer für diesen Zweck ausgewählten Expertengruppe via E-Mail zur Beurteilung folgender Aspekte vorgelegt:

- Verständlichkeit der Fragen

- Aufbau und Struktur des Formulars

- Praxistauglichkeit des Klassifikationssystem

- Benötigte Zeit zum Ausfüllen des Formulars

Die Expertengruppe umfasste eine Person aus dem Prozess- und Qualitätsmanagement der Spitex, drei bei der
Spitex im Pflegebereich tätige Personen, zwei Spitalapotheker, vier Offizinapotheker und eine Person aus der Pharmacovigilance (Systematische Überwachung von Arzneimitteln nach deren offizieller Marktzulassung).

Das Formular wurde anhand der Rückmeldungen adaptiert und anschließend in einem Pilotversuch in der Praxis getestet.

\section{Pilotierung des CIRS-Formulars}

Das CIRS-Formular wurde während eines Monats bei sieben von elf Teams der Spitex Stadt Luzern pilotiert. Ein Team umfasst 15-20 Mitarbeitende im Bereich Pflege. Die zum Zeitpunkt des Piloten in den Medikationsprozess involvierten Mitarbeitenden wurden mittels einer kurzen schriftlichen Einführung im Format einer DIN A4-Seite mit den Eigenheiten eines CIRS und dem Ablauf der Einführung vertraut gemacht. Sie wurden aufgefordert, sowohl Fehler wie auch Beinahe-Fehler, die als Fehler ohne Schädigung des Klienten definiert wurden, zu melden. Die direkten Vorgesetzten stellten sicher, dass alle in den Medikationsprozess involvierten Mitarbeitenden von der schriftlichen Einführung Kenntnis hatten. Von den Vorgesetzten wurden die Mitarbeitenden zudem wöchentlich aufgefordert, alle Ereignisse und Beinahe-Ereignisse mittels des Formulars in anonymisierter Form zu melden.

Die qualitative und quantitative Auswertung der Meldungen fand unabhängig durch zwei Prüf-Personen statt. Ein besonderes Augenmerk wurde auf die Kategorisierung gerichtet: Die Kategorisierung wurde entsprechend den Angaben der meldenden Person (subjektive Einschätzung) belassen, wenn die beiden Prüf-Personen unabhängig voneinander die Zuteilung nachvollziehen konnten. Ansonsten wurde die Kategorisierung im Konsens angepasst.

Zusätzlich wurden die Mitarbeitenden mittels einer elektronischen Umfrage zur Verständlichkeit des CIRS, zum Ablauf des CIRS (u.a. Zeitaufwand) und zu ihrem Umgang mit dem CIRS (u.a. Bedeutung der Anonymität) befragt. Eine Rückmeldung war obligatorisch für eine Teamleitung oder fallführende Mitarbeiterin aus jedem teilnehmenden Team ( $\mathrm{n}=11$ Personen). Weitere Rückmeldungen wurden ermutigt, insbesondere von Mitarbeitenden, welche während des Pilots eine Meldung im CIRS erfassten, waren jedoch freiwillig.

\section{Implementierungsstrategien}

Für die Implementierung und Etablierung des CIRS im Team der Spitex Stadt Luzern wurden verschiedene Strategien erarbeitet:

- Standardisierte Arbeitsanweisung zum Umgang mit dem Spitex-eigenen CIRS, die an allen Teamstandorten in gedruckter und elektronischer Form vorliegt. Die Arbeitsanweisung umfasst sechs DIN A4-Seiten, aufgebaut aus den folgenden Kapiteln: Ziel und Zweck, rechtliche Grundlagen, Definitionen (inkl. Fehler und Beinahe-Fehler), zu erfassende Meldungen, Dokumentation, Bearbeitung der Meldungen - CIRS-Gruppe, Rückmeldungen an die Mitarbeitenden, Archivierung, Schulungen. 
- Schulungsunterlagen in Form einer PowerPoint ${ }^{{ }_{-}}$ Präsentation für neue Mitarbeitende. Die Schulungsunterlagen umfassen einen Foliensatz von 30 Seiten, der Schulungsaufwand beträgt 30-60 Minuten. Es wird empfohlen, die Dokumentation schriftlich abzugeben.

- Gründung einer interdisziplinären CIRS-Gruppe zur regelmäßigen Evaluation der CIRS-Meldungen. Die CIRSGruppe umfasst die folgenden Fachpersonen: eine Person aus dem Qualitätsmanagement, zwei Teamleitungen, einen fallführenden Mitarbeiter, eine diplomierte Pflegefachperson, einen Fachangestellten Gesundheit und eine Person aus der Pflege. Als externe Experten können der Kontaktarzt der Spitex sowie der Apotheker, der mit der Spitex zusammenarbeitet, hinzugezogen werden. Die Gruppe trifft sich alle zwei Monate zur gründlichen Analyse der gemeldeten Ereignisse.

- Entwicklung des Publikationsorgans «Frühwarnung»eine regelmäßig erscheinende Team-Kurzinformation zu Ergebnissen aus dem CIRS mit konkreten Verbesserungsvorschlägen.

Die Auswertungen des CIRS werden alle zwei Monate als Teil des Spitexpress (interne Spitex-Zeitung) nach den CIRS-Gruppensitzungen als Frühwarnungen verschickt. Sie beinhalten eine statistische Auswertung und Zusammenfassung der wichtigsten kritischen Ereignisse sowie die Darstellung eines wichtigen Fallbeispiels inklusive Lösungsstrategien, neuen Vorschriften und Änderungen im Arbeitsprozess, um weitere ähnliche Vorkommnisse zu verhindern.

Diese Publikationen werden auch durch die Teamleitungen an den Stellwänden in den Teamstationen aufgehängt. Die Mitarbeiter können auf einem separaten Blatt neben den aushängenden Frühwarnungen eigene Lösungs- und Verbesserungsvorschläge notieren. Erachtet die CIRS-Gruppe eine CIRS-Meldung als besonders schwerwiegend respektive relevant, wird eine dringende Warnung sofort per E-Mail durch dieselbe Gruppe an alle Mitarbeitenden versendet.

\section{Ergebnisse}

\section{Entwicklung des CIRS-Formulars}

Das Formular wurde in folgende Teile gegliedert:

- Angaben zur meldenden Person (fakultativ)

- Informationen zum Patienten

- Protokoll des Ereignisses

- Klassifikation medikationsassoziierter Probleme

- Angaben zu den Ursachen

- Ergriffene Sofortmaßnahmen

- Vorschläge zur zukünftigen Verhinderung ähnlicher Ereignisse

- Einschätzung des Schweregrads

- Bemerkungen
Das Formular wurde so konstruiert, dass die Anonymität der meldenden Person sowie des Patienten gewährleistet wird. Für die meldende Person besteht jedoch die Möglichkeit, freiwillig ihren Namen anzugeben für allfällige Rückfragen bei der Auswertung.

Die Ereignisbeschreibung erfolgt im Freitextformat.

Als Basis für die Klassifizierung medikationsassoziierter Probleme diente die PCNE-Klassifizierung der Pharmaceutical Care Network Europe Foundation (PCNE, 2006), die jedoch mit Hilfe der Literatur an den ambulanten Bereich angepasst wurde. (Audette, Triller, Hamilton \& Briceland, 2002; WHO, 2009; van Mil, Westerlund, Hersberger \& Schaefer, 2004)

Der Pretest zeigte insbesondere Optimierungspotenzial in den folgenden Bereichen:

- Übersichtlichere Gliederung und Nummerierung der Hauptbereiche des Formulars für eine logische Gesamtstruktur

- Logisch-hierarchische Anordnung der vorgegebenen Antwortmöglichkeiten

- Verständlichkeit der Antwortmöglichkeiten adaptiert an den Pflegeberuf, da das CIRS bei der Spitex zum aktuellen Zeitpunkt ausschließlich durch Pflegefachpersonen ausgefüllt wird und sich auf ihre spezifischen Tätigkeiten bezieht.

\section{Pilotierung des CIRS-Formulars}

Das entsprechend dem Pre-Test überarbeitete Formular (siehe Abbildung 1) wurde während vier Wochen pilotiert. Während des Pilot-Monats wurden 43 CIRS-Formulare retourniert. In der ersten Woche nach Lancierung des Pilots wurden $50 \%$ der Formulare (21) ausgefüllt. In $76 \%$ der Meldungen war die meldende Person der Entdecker des Ereignisses.

Insgesamt sechs Formulare (14\%) waren unvollständig: Bei drei Meldungen fehlte die Angabe der Berufsgruppe der meldenden Person. Bei drei Formularen fehlten mehrere Kategorie-Zuteilungen; bei zweien davon wurde zusätzlich die Einteilung des Schweregrads unterlassen.

In insgesamt $34 \%$ der Meldungen waren eindeutige Rückschlüsse auf die meldende Person möglich. Die Möglichkeit anonym zu melden, wurde jedoch in einer nachfolgenden Befragung durch sechs von neun Personen als wichtig und durch zwei als sehr wichtig beurteilt. Trotzdem würden fünf von neun Personen ziemlich wahrscheinlich und sechs ganz sicher auch Meldung erstatten, wenn das CIRS nicht anonym wäre.

Die von CIRS-Meldungen betroffenen Klienten waren im Durchschnitt 78,7 \pm 15,3 Jahre alt; 74 \% (32) waren Frauen.

Bei drei Meldungen fehlten mehrere Kategorie-Zuteilungen, die basierend auf der Freitextbeschreibung nicht ergänzt werden konnten, weshalb nur 40 Meldungen bezüglich betroffenem Teilprozess aufgeschlüsselt werden konnten. In $54 \%$ (22 Meldungen von 40) wurden Fehler gemeldet, die sich beim Richten (inkl. Liefern) der Medi- 


\section{Formular zur Meldung eines kritischen Ereignisses CIRS - Critical Incident Reporting System}

Das Formular dient der Qualitätsverbesserung durch Erfassen von kritischen Ereignissen (Ereignisse umfassen Fehler und Beinahe-Fehler von Material, Planung, Bestellung, Medikation, Pflege). Es kann anonym ausgefüllt werden und die Meldung hat keine Konsequenzen für den Melder. In bestimmten Abständen werden Rückmeldungen mit Lösungsvorschlägen kommuniziert.

\begin{tabular}{|l|l|}
\hline A) Meldende Person \\
\hline Name (freiwillig): & Team: \\
\hline Bezug zum Ereignis: $\square$ verursacht $\quad \square$ entdeckt $\square$ informiert durch Dritte \\
\hline
\end{tabular}

B) Informationen zum Patienten

\begin{tabular}{l|l|} 
Jahrgang: & Geschlecht: $\square$ männlich $\quad \square$ weiblich \\
\hline
\end{tabular}

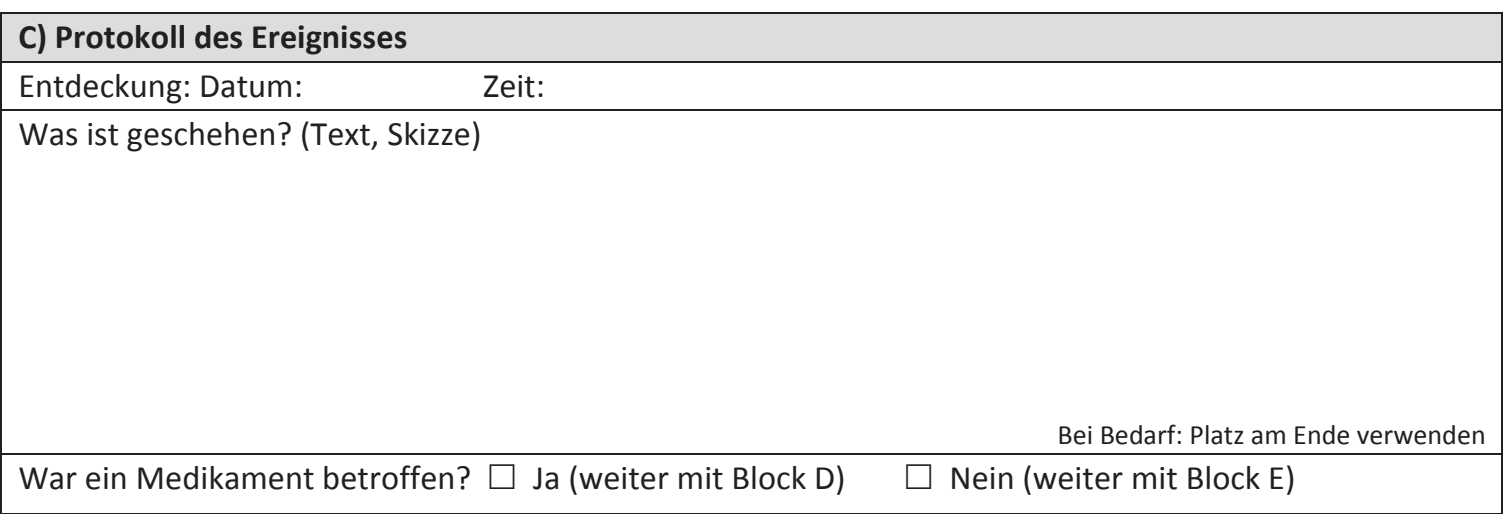

\section{D) Beschreibung des arzneimittelbezogenen Problems}

Womit stand das Ereignis im Zusammenhang? (Mehrfachantworten möglich)

\section{D1) Verschreibung:}

$\square$ Verschreibung nicht vorhanden Verschreibung unvollständig Verschreibung fehlerhaft Andere:

\section{D2) Richten:}

$\square$ Medikament nicht vorhanden (Nachschubproblem) Medikament nicht gerichtet

$\square$ Falsches Medikament gerichtet

Medikament ohne Verordnung gerichtet

Falsche Dosierung gerichtet

Zum falschen Zeitpunkt gerichtet

Medikament verabreicht nach Verfall

Probleme mit Dispenser/Dosett

Fehlende Doppelkontrolle

D3) Abgabe / Einnahme / Verabreichung

Unerwünschte Wirkung

Medikament nicht eingenommen

$\square$ Falsches Medikament eingenommen

Falsche Dosis eingenommen

Einnahme zu einem falschen Zeitpunkt

Selbstmedikation durch Patienten

Kontrolle fehlerhaft

Bei einer nicht regulären Kontrolle entdeckt

\section{D4) Dokumentation:}

$\square$ Fehlende/Unvollständige/Falsche Übertragung auf Medikamentenblatt

$\square$ Fehlende Dokumentation bei der Verabreichung von Reservemedikation

$\square$ Fehlende Massnahmenplanung in der PPL (z.B. Verweigerung der Einnahme, verlangt mehr Schlafmittel)

$\square$ Unklare PPL

Andere: 


\section{Formular zur Meldung eines kritischen Ereignisses CIRS - Critical Incident Reporting System}

\begin{tabular}{|c|c|}
\hline \multicolumn{2}{|l|}{ E) Wo lagen die möglichen Ursachen? (Mehrfachantworten möglich) } \\
\hline E1) Räumlichkeiten & E2) Kommunikation mangelhaft \\
\hline$\quad$ Platzverhältnisse zu gering & $\quad$ Arzt-Patient \\
$\square$ Schlechte Lichtverhältnisse & Arzt - Spitex \\
$\square$ Ungenügende Sauberkeit & $\square$ Apotheke - Spitex \\
& $\square$ Patient - Spitex \\
& $\square$ Spitex - Spitex \\
\hline E3) Arbeit & E4) weitere Ursachen \\
$\square \quad$ Zeitdruck, Stress & $\square \quad$ Einnahmebeschwerden (z.B. Schlucken, Zittern) \\
$\square$ Überarbeitung & $\square$ Schlechte Lesbarkeit \\
$\square$ Unaufmerksamkeit & $\square$ Fehlende/Unvollständige/Falsche Übertra- \\
$\square$ Ablenkung durch............................. & gung auf Medikamentenblatt \\
$\square$ Ausbildung, Training & $\square \quad$ Kontrolle nicht korrekt \\
& $\square \quad$ Unklar \\
& $\square \quad$ Andere: ............................. \\
\hline
\end{tabular}

\section{F) Welche Sofortmassnahmen wurden ergriffen?}

G) Vorschläge, damit sich dieses Ereignis in Zukunft nicht wiederholen wird:

\begin{tabular}{|c|c|c|c|c|c|}
\cline { 3 - 5 } \multicolumn{2}{c}{} & \multicolumn{3}{c|}{ Schweregrad } & geringfügig \\
\cline { 3 - 6 } \multicolumn{2}{c|}{$\begin{array}{c}\text { Auftretungswahr- } \\
\text { scheinlichkeit }\end{array}$} & Schwerwiegend & bedeutend & mässig & \\
\cline { 2 - 6 } & Gelegentlich & & & & \\
\cline { 2 - 6 } & Selten & & & & \\
\cline { 2 - 5 } & Sehr selten & & & & \\
\hline
\end{tabular}

Bemerkungen 
kamente ereigneten. Die weitere Fehlerverteilung bezogen auf den betroffenen Prozessschritt ist in Abbildung 2 ersichtlich.

Der meistgenannte Fehler beim Richten war die Unterlassung (30\%), gefolgt von einer falschen Dosierung (17,5\%) und dem falschen Zeitpunkt (15\%).

Insgesamt $12,2 \%$ der Meldungen betrafen die Verschreibung, wobei insbesondere der Aspekt der fehlenden schriftlichen Verordnung genannt wurde $(66,7 \%)$.

Als Hauptursache für Fehler gaben die meldenden Personen die Arbeitsbedingungen an (37,9\%), wobei insbesondere Unaufmerksamkeit (68,2\%), Zeitdruck (22,7\%) und Unterbrechungen durch den Klienten (9,1 \%) der Auslöser waren.

Als zweithäufigste Ursache wurde mangelhafte Kommunikation genannt (36,2\%). Die Kommunikation wurde sowohl innerhalb der Spitex $(38,1 \%)$ wie auch mit den ärztlichen Partnern (33,3\%) als ungenügend beurteilt.

Als Sofortmaßnahme wurde in den meisten Fällen die Medikation neu gerichtet (31,9\%). In 23,4\% der Fälle wurde eine zusätzliche Fachperson konsultiert (Teamleitung Spitex, Hausarzt, Klient).

Der Schweregrad wurde in $95 \%$ der Fälle durch die meldende Person geschätzt, in $5 \%$ durch die beiden beurteilenden Fachexperten ergänzt. Es wurden keine Re-Kategorisierungen des Schweregrads vorgenommen. In $45 \%$ der Fälle erreichte das Ereignis den Klienten nicht, in 47,5\% erreichte das Ereignis den Klienten, hatte jedoch keine erkennbaren Konsequenzen, in einem Fall erlitt ein Klient eine temporäre gesundheitliche Beeinträchtigung.

Die Umfrage zur Beurteilung des CIRS war während zwei Wochen online verfügbar. Es nahmen 17 Personen an der Umfrage teil; 13 Frauen und vier Männer. Dabei handelte es sich um fünf Teamleitungen, neun fallführende Mitarbeiterinnen, eine Pflegefachperson, eine Pflegehilfe sowie eine Person mit unbekannter Funktion. Je vier Personen arbeiteten in einem Pensum von $100 \%$ resp. $80 \%$, je 3 Personen mit $90 \%$ resp. $40 \%$, die übrigen mit $60 \%$ (zwei Personen) und $70 \%$ (eine Person). Vier Personen waren seit mehr als zehn Jahren bei der Spitex tätig, drei Personen seit 6-10 Jahren, neun Personen seit 1-5 Jahren und eine Person seit weniger als einem Jahr.

Die Teilnehmenden gaben mit 57,1 \% einen Zeitbedarf für das Ausfüllen des Formulars von weniger als zehn Minuten an, die restlichen $42,9 \%$ benötigten zwischen zehn und 20 Minuten.

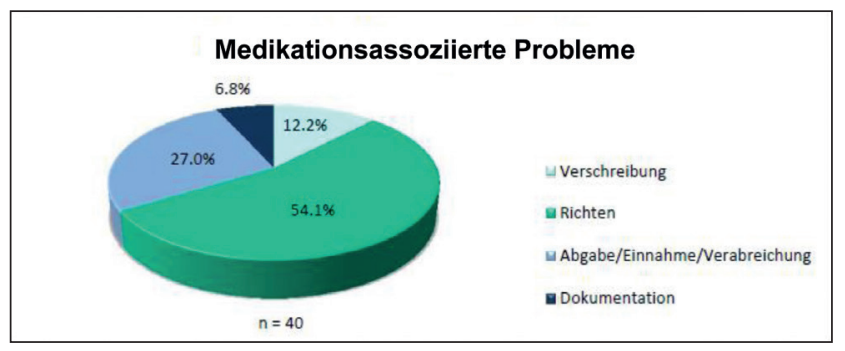

Abbildung 2. Prozessverteilung der gemeldeten medikationsassoziierten Probleme ( $n=40$ Meldungen, 3 Meldungen ohne Angaben zum betroffenen Prozessschritt).
Alle Teilnehmenden waren mit dem Aufbau des Formulars und der Übersichtlichkeit zufrieden oder sehr zufrieden. Die Platzverhältnisse für die Freitextangaben waren für $81,8 \%$ ausreichend. Lediglich eine Person hatte inhaltlich Verständnisschwierigkeiten.

\section{Implementierungsstrategien}

Die Arbeitsanweisung wurde entsprechend dem Format des bestehenden Qualitätsmanagementsystems der Spitex Stadt Luzern erstellt.

Die Schulungsunterlagen in Form einer PowerPointPräsentation beinhalteten einen allgemeinen Teil zum Thema CIRS, eine Anleitung zum Umgang mit dem neuen CIRS-Formular in der Spitex Stadt Luzern (Fehlerkultur, was soll gemeldet werden, Abgrenzung zum Beschwerdemanagement sowie Nennung konkreter Beispiele aus der Pilotphase).

Anschließend an den Piloten wurde eine CIRS-Gruppe implementiert, welche regelmäßig eine Review der Auswertung der gemeldeten Fälle vornimmt, die wichtigsten Meldungen als Grundlage für Verbesserungsmaßnahmen aufarbeitet und den Mitarbeitenden zeitnah kommuniziert.

\section{Diskussion}

Die Verfügbarkeit von Literatur zu DRPs sowie zu Critical Incident Reporting Systemen im Home Care Bereich ist limitiert. Zusätzlich erschwerend bei der Erfassung und Auswertung von Zwischenfällen und Beinahe-Zwischenfällen ist die uneinheitliche Nomenklatur und Klassifizierung der DRPs.

Diese Arbeit ist unseres Wissens der erste Ansatz, ein systematisch entwickeltes CIRS-Formular mit Schwerpunkt DRPs im Home Care Bereich zu lancieren. Der Aufbau eines CIRS-Formulars basierend auf Vorlagen aus dem stationären Bereich sowie einer gründlichen Test- und Pilotphase hat zu einem Formular geführt, das von den Mitarbeitenden gut akzeptiert wurde und eine Erfassung und ein Trending von DRPs mit begrenztem zeitlichen Aufwand pro Meldung erlaubte. Bei einer weiteren Optimierung des CIRS-Formulars sollte ein direkteres Feedbackvorgehen ins Auge gefasst werden, um eine höhere Rücklaufquote der Mitarbeiterbefragung sicherzustellen.

Obwohl in der Literatur zur Erhöhung der Meldungsfrequenz ein anonymer Ansatz propagiert wird, erlaubt eine nicht-anonyme Meldung die Generierung von Zusatzdaten (Mahajan, 2010). Deshalb wird der meldenden Person die Identifikationsmöglichkeit offen gelassen. In $34 \%$ der Meldungen hat die meldende Person freiwillig auf die Anonymität verzichtet. Im Falle eines nicht-anonymen CIRS sollte den Mitarbeitenden in der begleitenden Arbeitsanweisung zugesichert werden, dass bei Offenlegung der Identität keine Nachteile entstehen und dies lediglich der Klärung der Meldung dient, um die Patientensicherheit zu erhöhen. 
Das ausführlich strukturierte Format des Formulars wurde mit der Absicht gewählt, möglichst detaillierte Informationen von der meldenden Person zu erhalten, da bei anonymer Meldung keine Rückfragen möglich sind. Die Kategorisierung durch die meldende Person wurde jeweils durch zwei unabhängige Fachpersonen überprüft, um eine einheitliche Auswertung zu garantieren. War die Kategorisierung durch die meldende Person nachvollziehbar, wurde diese beibehalten unter der Annahme, dass die meldende Person näher am Ereignis ist. Die Einteilung durch die meldende Person wurde somit prioritär behandelt. Die Autoren sind von der Annahme ausgegangen, dass sich die Prozesse im Home Care Bereich von den Prozessen im stationären Bereich unterscheiden und deshalb ein Home Care-spezifisches CIRS-Formular sinnvoll ist. Die neu entwickelten Home Care-spezifischen Rubriken zum betroffenen Prozess (Verschreibung nicht vorhanden) und zu den Ursachen (Räumlichkeiten, Unterbrechungen durch den Klienten, Kommunikation) wurden von den meldenden Personen häufig benutzt und scheinen daher sinnvoll und gerechtfertigt.

Aufgrund der Freiwilligkeit von Meldungen in einem Critical Incident Reporting System ist das Verhältnis der Anzahl gemeldeter Fehler zur Anzahl der Fehler, die sich tatsächlich ereignet haben, unklar. Eine längere Beobachtungszeitspanne könnte vorteilhaft sein, um das Meldeverhalten und die Anzahl gemeldeter Fehler besser einschätzen zu können.

Die Analyse der in der Pilotphase gemeldeten CIRS-Fälle zeigte, dass die meisten gemeldeten medikationsassoziierten Fehler das Richten der Medikamente betreffen. Dies wird auch dadurch reflektiert, dass Fehler häufig durch die beobachtende Person beim Kontrollieren der gerichteten Medikamente entdeckt werden. Dieses Resultat wird zudem bestätigt durch Schätzungen, wonach bis zu $50 \%$ der Medikationsfehler im Rahmen dieser Tätigkeiten geschehen (Bates et al., 1995). Bis zu $5 \%$ der Medikamente insgesamt werden fehlerhaft gerichtet. (Zieglmeier, Frenger, Amann, Oehring \& Grosshans, 1998; Altersberger, Blanché-Ganter, Ilg \& Keck-Benz, 2004; Schreck, Buxtorf, Plagge \& Deuster, 2009)

Obwohl die Hälfte der Fachpersonen mit den örtlichen Bedingungen beim Richten der Medikamente zufrieden ist, wird in der Auswertung des CIRS das Ergebnis der Umfrage bestätigt, dass die Störungen durch die Klienten signifikant zur Fehlerentstehung beitragen können.

Die Beurteilung der Verwendung eines strukturierten Meldeformulars durch die Mitarbeitenden war positiv. Der zeitliche Aufwand wurde als vertretbar beurteilt, wobei ein elektronisches Format zeitlich vorteilhaft sowohl für die Erfassung wie auch für die Auswertung sein könnte und dadurch die Melde-Motivation möglicherweise erhöht werden könnte. Flankierende Maßnahmen zur Implementierung, wie Schulungen und regelmäßige Personalinformation $\mathrm{zu}$ den CIRS-Ergebnissen, sind wichtig für den qualitativen und quantitativen Rücklauf der Meldungen.

Eine Ausweitung des Formulars, welches eine Erfassung von kritischen Ereignissen erlaubt, die nicht ausschließlich mit der Medikation in Zusammenhang stehen, ist geplant, um die Prozessqualität gesamthaft zu optimieren.

Wünschenswert ist außerdem, die Fehlerproblematik an den Schnittstellen mit den Zuweisern (niedergelassene Ärzte, Spitäler) genauer zu analysieren und in einem interprofessionellen Team unter Einbezug der Ärzte und weiterer involvierter Fachpersonen (u.a. Case Manager, Sozialarbeiter, Apotheker) zu optimieren.

\section{Schlussfolgerung}

Der Einsatz eines standardisierten, manuellen CIRS-Formulars ist einfach und kostengünstig. Die Pilotierung zeigte, dass die häufigsten Meldungen das Richten (inkl. Liefern) der Medikamente betrafen. Dies ist ein kritischer Prozess, welcher im Rahmen der Qualitätssicherung in Ergänzung zu einem CIRS weitergehende Kontrollen erfordert. Als nächster Schritt sollte der vorliegende Entwurf nun in weiteren Studien auch in anderen Regionen erprobt werden. Zur Zeitersparnis bei Erfassung und Auswertung sollte ein elektronisches Format des CIRS-Formulars in Betracht gezogen werden. Dies würde auch eine nationale Vernetzung vereinfachen.

\section{Auftraggeber/Förderung}

Das Projekt wurde im Rahmen einer Masterarbeit an der Universität Basel durchgeführt. Es bestand keinerlei Auftrag oder Förderung durch externe Parteien. Die Autoren haben keinerlei Intressenskonflikte. Die Autoren bedanken sich herzlich bei den Mitarbeitenden der Spitex Stadt Luzern für die engagierte Beteiligung am Projekt.

\section{Beiträge der einzelnen Autor(inn)en}

Substanzieller Beitrag zu Konzeption oder Design der Arbeit: CM, SL, EK, BH, KH

Substanzieller Beitrag zu Konzeption oder Design der Arbeit: EK, CM, BH, KH, SL

Manuskripterstellung: CM, EK, KH, BH, SL

Einschlägige kritische Überarbeitung des Manuskripts: CM, EK, KH, BH, SL

Genehmigung der letzten Version des Manuskripts: CM, EK, KH, BH, SL

Übernahme der Verantwortung für das gesamte Manuskript: CM, KH

\section{Literatur}

Ahrens, J. (2003). Combatting medication errors in home health. Caring, 22, $56-9$ 
Akers, W. S.; Flynn, J. D.; Davis, G. A.; Green, A. E.; Winstead, P. S.; Strobel, G. (2004). Prolonged cardiac repolarization after tacrolimus and haloperidol administration in the critically ill patient. Pharmacotherapy 24, 404-408.

Altersberger. I.; Blanché-Ganter, E.; Ilg, A.; Keck-Benz, L. (2004). Aufbau und Einführung eines Sicherheitsmanagements zur Vermeidung von Medikationsirrtümern. AdkA Wissenschaftlicher Kongress Weimar.

Audette, C. M.; Triller, D. M.; Hamilton, R.; Briceland, L. L. (2002). Classifying drug-related problems in home care. American Journal of Health-System Pharmacy, 59, 2407 - 2409.

Bates, D. W.; Cullen, D. J.; Laird, N. et al. (1995). Incidence of adverse drug events and potential adverse drug events. Implications for prevention. ADE Prevention Study Group. JAMA, 274, 29 - 34

Bond, C. A.; Raehl, C. L. (2007). Clinical pharmacy services, pharmacy staffing, and hospital mortality rates. Pharmacotherapy, 27, $481-493$.

Classen, D. C.; Metzger, J. (2003). Improving medication safety: the measurement conundrum and where to start. Internatioal Journal of Quality in Healthcare, 15 Suppl 1, i41-i7.

Fernald, D. H.; Pace, W. D.; Harris, D. M.; West, D. R.; Main, D. S.; Westfall, J. M. (2004). Event reporting to a primary care patient safety reporting system: a report from the ASIPS collaborative. Annals of family medicine, 2, 327-332.

Fialova, D.; Topinkova, E.; Gambassi, G. et al. (2005). Potentially inappropriate medication use among elderly home care patients in Europe. JAMA, 293, $1348-1358$.

Fiss, T.; Dreier, A.; Meinke, C.; van den Berg, N.; Ritter, C. A.; Hoffmann, W. (2011). Frequency of inappropriate drugs in primary care: analysis of a sample of immobile patients who received periodic home visits. Age Ageing, 40, 66 - 73.

Flynn, E. A.; Barker, K. N.; Pepper, G. A.; Bates, D. W.; Mikeal, R. L. (2002). Comparison of methods for detecting medication errors in 36 hospitals and skilled-nursing facilities. American Journal of Health System Pharmacy, 59, 436-46.

Katz, R. I.; Lagasse, R. S. (2000). Factors influencing the reporting of adverse perioperative outcomes to a quality management program. Anesthesia \& Analgesia, 90, 344-350.

Mahajan, R. P. (2010). Critical incident reporting and learning. British Journal of Anaesthesia, 105, $69-75$.

McGraw, C.; Topping, C. (2011). The District Nursing Clinical Error Reduction Programme. British Journal of Community Nursing, $16,35-40$.

Meredith, S.; Feldman, P. H.; Frey, D. et al. (2001). Possible medication errors in home healthcare patients. Journal of American Geriatrics Society, 49, $719-724$.

Meyer-Massetti, C.; Conen, D. (2012). Assessment, frequency, causes, and prevention of medication errors - a critical analysis. Therapeutische Umschau Revue therapeutique, 69, 347 - 52.

Meyer-Massetti, C.; Kaiser, E.; Hedinger-Grogg, B.; Luterbacher, S.; Hersberger, K. (2012). Medication safety in the home care setting: error-prone process steps. Pflege, 25, 261-269.

PCNE classification for drug related problems. (2006). www.pcne. $\mathrm{org} / \mathrm{sig} / \mathrm{drp} /$ documents/PCNE\%20classification\%20V6-2.pdf [10.3.2013]

Roughead, E. E.; Semple, S. J.; Rosenfeld, E. (2016). The extent of medication errors and adverse drug reactions throughout the patient journey in acute care in Australia. International Journal of Evidence-based Healthcare, 4.3.2016 Epub ahead of print.

Schreck, S.; Buxtorf, R.; Plagge, H.; Deuster, S. (2008). Der Medikationsprozess am Beispiel einer geriatrischen Station -
Das Arzneimittel von der Verordnung bis zur Einnahme. www. unispital-basel.ch/fileadmin/unispitalbaselch/Bereiche/ Querschnittsfunktionen/Spital-Pharmazie/poster_fps_projekt.pdf [06.06.2016]

Shojania, K. G. (2008). The frustrating case of incident-reporting systems. Quality \& Safty in Health Care, 17, 400-402.

Spitex Stadt Luzern. (2013). Jahresbericht 2013. www.spitex-luzern.ch/fileadmin/pdf/publikationen/spitex_jb_2013_ansicht. pdf [28.8.2011]

Tam, K. W.; Kwok, K. H.; Fan, Y. M. et al. (2008). Detection and prevention of medication misadventures in general practice. International Journal of Quality in Health Care, 20, 192-199.

van Mil, J. W.; Westerlund, L. O.; Hersberger, K. E.; Schaefer, M. A. (2004). Drug-related problem classification systems. Annals of Pharmacotherapy, 38, 859 - 867.

WHO (2005). WHO draft guidelines for adverse event reporting and learning systems - from information to action. www.who.int/ patientsafety [06.06.2016].

WHO (2009). The conceptual framework for the international classification for patient safety 1/1/2009.

Zieglmeier, M. Frenger, J.; Amann, S.; Oehring, U.; Grosshans, R. (1998). Arzneimittelsicherheit beim Stellen fester oraler Arzneiformen. AdkA Wissenschaftlicher Kongress 1998.

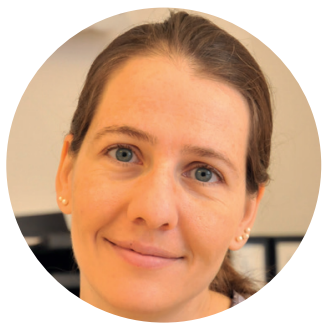

Dr. phil. II Carla Meyer-Massetti Universität Basel

Klinische Pharmazie \& Epidemiologie

Spitalstrasse 26

4031 Basel

Schweiz

carla.meyer@unibas.ch

\section{Was war die größte Herausforderung bei Ihrer Studie?}

Die limitierte Datenlage zur Medikationssicherheit im Home Care Bereich.

\section{Was wünschen Sie sich bezüglich der Thematik für die Zukunft?}

Entwicklung von evidence-based Interventionen zur Optimierung der Medikationssicherheit im Home Care Bereich; Optimierung und Standardisierung der interprofessionellen Zusammenarbeit.

\section{Was empfehlen Sie den LeserInnen zum Weiterlesen/Vertiefen?}

Projektinformationen zu «Transition of Care» https://clinicalpharmacy. ucsf.edu/(Department Klinische Pharmazie, University of California, San Francisco).

Manuskripteingang: 02.12.2015

Manuskript angenommen:17.05.2016

Veröffentlicht online: 06.07.2016 